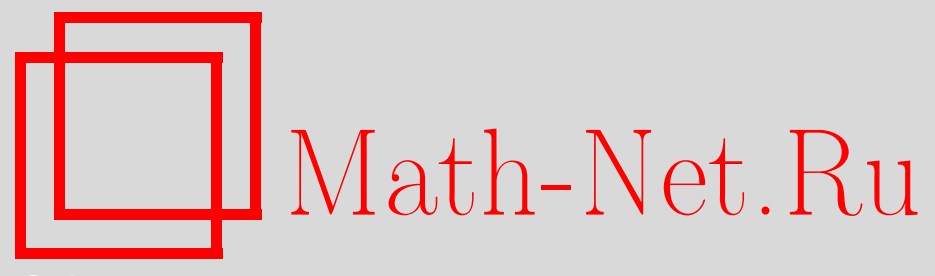

В. П. Радченко, А. В. Нехожин, Математическое моделирование деформирования армированной бедренной кости при длительных статических нагрузках, Вестн. Сам. гос. техн. ун-та. Сер. Физ.-мат. науки, 2015, номер 4, 768-784

DOI: https://doi.org/10.14498/vsgtu1441

Использование Общероссийского математического портала MathNet.Ru подразумевает, что вы прочитали и согласны с пользовательским соглашением

http://www.mathnet.ru/rus/agreement

Параметры загрузки:

IP : 52.87 .193 .239

26 апреля 2023 г., 12:06:34

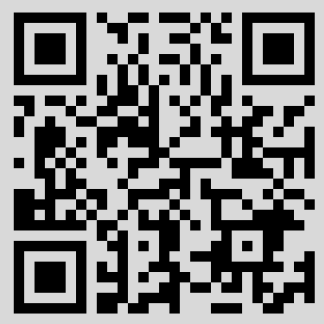


УДК 539.376 + 612.76:616.71-001.5

\title{
МАТЕМАТИЧЕСКОЕ МОДЕЛИРОВАНИЕ ДЕФОРМИРОВАНИЯ АРМИРОВАННОЙ БЕДРЕННОЙ КОСТИ ПРИ ДЛИТЕЛЬНЫХ СТАТИЧЕСКИХ НАГРУЗКАХ
}

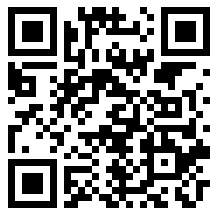

\author{
В. П. Радченко, А. В. Нехожин
}

Самарский государственный технический университет,

Россия, 443100, Самара, ул. Молодогвардейская, 244.

\begin{abstract}
Аннотация
Предложена двухслойная математическая модель шейки бедренной кости человека, армированной имплантатами различной конструкции, для моделирования напряжённо-деформированного состояния, возникающего при хирургической методике профилактики переломов шейки бедра путём принудительного введения металлических имплантатов. Приведены спроектированные конструкции имплантатов. Разработаны методика и программное обеспечение для геометрического моделирования бедренной кости с внедрёнными имплантатами. Сформулированы постановки новых краевых задач для оценки кинетики напряжённодеформированного состояния в армированной и неармированной шейке бедра при длительных статических нагрузках, соответствующих хождению человека, в условиях ползучести. Приведены эффективные упругие характеристики для компактной и губчатой костных тканей, силовые и кинематические граничные условия задач. Построена феноменологическая модель ползучести компактной костной ткани, разработана методика идентификации параметров и выполнена проверка её адекватности экспериментальным данным. На основе метода конечных элементов разработан численный метод решения поставленных краевых задач на уровне макромеханики сплошных сред. Многочисленные вариативные расчёты позволили выработать рекомендации по рациональному позиционированию имплантатов для максимального снижения концентрации напряжений. Выполненный анализ показал, что происходит существенная релаксация напряжений в наиболее нагруженных областях вследствие ползучести, причём в армированной шейке бедра она происходит более интенсивно, чем в неармированной. Так, для имплантата «спица + спица» при длительности нагружения в течение 1 года при естественных нагрузках, соответствующих хождению человека, напряжённость в наиболее нагруженной области шейки бедра вследствие ползучести снижается на 49 \% по отношению к напряжённости в начальный момент приложения нагрузки. Установлено, что временная составляющая (длительная стационарная нагрузка) не ухудшает положительный
\end{abstract}

(C) 2015 Самарский государственный технический университет.

\section{Образец для цитирования}

Р ад ченко В. П. Нехо жин А. В. Математическое моделирование деформирования армированной бедренной кости при длительных статических нагрузках // Becmн. Caм. гос. техн. ун-та. Сер. Физ.-мат. науки, 2015. Т. 19, № 4. С. 768-784. doi: 10.14498/vsgtu1441.

\section{Сведения об авторах}

Владимир Павлович Радченко (д.ф.-м.н., проф.; radch@samgtu.ru; автор, ведущий переписку), заведующий кафедрой, каф. прикладной математики и информатики.

Анатолий Вадимович Нехожин (stswoon@yandex.ru), аспирант, каф. прикладной математики и информатики. 
эффект от снижения концентрации напряжений за счёт армирования шейки бедра, что является позитивным моментом с точки зрения медицинской практики.

Ключевые слова: бедренная кость, имплантат, шейка бедра, геометрическое моделирование, метод конечных элементов, ползучесть, напряжённо-деформированное состояние, релаксация.

doi: http://dx.doi.org/10.14498/vsgtu1441

Введение. Проблема повышения прочности является центральной не только для традиционных конструкционных материалов и элементов конструкций из них, но и для биокомпозитных материалов, например, костной ткани. Наиболее проблемной природной биокомпозитной «конструкцией» является шейка бедра, перелом которой приводит к крайне тяжелым последствиям. Увеличение характеристик прочности костной ткани решается различными способами, один из которых заключается в превентивном армировании металлическими имплантатами бедренной кости для снижения концентрации напряжений. В частности, в работе [1] предложена хирургическая методика профилактики (предупреждения) переломов шейки бедренной кости путём принудительного введения металлических имплантатов. Потенциальные перспективы применения данного подхода с точки зрения медицины очевидны и заключаются в снижении вероятности перелома и стоимости лечения по сравнению с процедурой сращивания сломанной костной ткани, простоте операции и т. д. Однако сертификация этого подхода требует комплексного подхода как со стороны медицинской науки, так и со стороны математиков, механиков и специалистов в области информационных технологий. Это обусловило начало комплексных исследований по оценке напряжённо-деформированного состояния армированной костной ткани. Так, в работах [2-4] предложены оригинальные конструкции металлических имплантатов для армирования шейки бедра (спица, спираль (штопор), винт-шнек), а в работе [5] предложена упрощенная однослойная модель армированной бедренной кости, состоящей лишь из прочной кортикальной её части. Выполненное моделирование влияния имплантатов на напряжённо-деформированное состояние шейки бедра для экстремальных условий (падение человека) дало положительные результаты в том смысле, что наблюдалось снижение концентрации напряжений в наиболее нагруженных областях более чем на $10 \%$ по сравнению с неармированной «конструкцией». Однако костная ткань - это достаточно сложный биокомпозиционный материал, и на уровне макромеханики сплошных сред часто рассматривают двухслойную модель, состоящую из кортикальной кости - внешний слой и губчатой кости (много менее прочной) - внутренний слой $[6,7]$, хотя и такое приближение является в определенном смысле достаточно грубым. В исследовании [8] на основании двухслойной модели разработан численный метод решения задачи оценки напряжённо-деформированного состояния армированной шейки бедра для кратковременного («мгновенного») нагружения. Выполненный анализ для разных схем армирования (спица сверху, спица снизу, спица + спица, спираль, спираль + спица, штопор) показал, что максимальное снижение уровня напряжений (для данного вида нагрузки) наблюдается для системы «кость - спица + спица» и составляет чуть более $12 \%$ по сравнению с неармированной шейкой. Таким образом, 
установлено, что учёт губчатой кости незначительно увеличивает величину снижения концентрации напряжений. Отметим также исследования, выполненные в работах $[9,10]$, в которых для биоманекенов экспериментальным путём установлено увеличение разрушающей нагрузки при двух видах нагружения - нагружение головки армированной шейки бедра вдоль оси бедренной кости и в перпендикулярном к ней направлении - от 27 до 93\% по отношению к неармированной костной ткани в зависимости от типа имплантата и схемы его позиционирования.

Отмеченные выше работы касались проблемы моделирования в условиях кратковременного («мгновенного») нагружения, при этом нагрузки соответствовали реальным, возникающим в экстремальных ситуациях, например при падении человека.

Однако костная ткань обладает явно выраженными реологическими деформациями, развивающимися во времени. Экспериментальные исследования ползучести костной ткани выполнены в работах [11-13], где приведены кривые стационарной ползучести при различных постоянных напряжениях. В работе [14] сделана попытка построить феноменологическую реологическую модель костной ткани на основе данных работ [11-13] с использованием обобщённой нелинейной модели Максвелла, но обобщить эту структурную модель на сложное напряжённое состояние проблематично, тем более использовать её при решении краевых задач. Отсюда возникает следующая задача: каким образом деформация ползучести скажется на кинетике напряжённодеформированного состояния в армированной бедренной кости? K сожалению, в настоящее время отсутствуют методы решения краевых задач для «конструкций» из биокомпозиционного материала-костной ткани в условиях ползучести как с учётом армирования, так и без него, разработка которых и является целью настоящей работы.

1. Геометрическое моделирование бедренной кости и имплантатов. Аналогично работе [8] рассматривается двухслойная математическая модель бедренной кости: внешний слой - кортикальная кость (компактная костная ткань), внутренний - губчатая кость (много менее прочная). Для построения геометрической модели бедренной кости использовалась её информационная модель проекта The Titanium Bone Project, полученная при помощи лазерного сканирования [15-17]. Однако эта информационная модель чрезмерно детализирована и её применение для численного решения краевых задач ползучести крайне проблематично. Поэтому необходимо выполнить упрощение этой модели. Для этого в настоящей работе предложен алгоритм построения геометрической объёмной модели, основные особенности которого следующие:

- использование алгоритма автоматического упрощения геометрии кости;

- значительное снижение времени построения геометрии кости;

- возможность регулировать точность построения геометрии.

В качестве алгоритма автоматического упрощения геометрии кости проекта The Titanium Bone Project ${ }^{1}$ использовался алгоритм Quadric Edge Collapse Decimation (QECD), который позволяет упростить модель без на-

\footnotetext{
${ }^{1}$ VRML-модель кости, полученная лазерным сканированием, находится на сайте проекта The Titanium Bone Project. Ссылка на VRML-модель кости, указанная в работе [8], больше не действительна.
} 
рушения топологии. Для этой цели выбран программный продукт MeshLab (http://meshlab.sourceforge.net/), который представляет собой набор различных фильтров для работы с объёмными моделями. Один из фильтров для этой программы и реализует необходимый алгоритм. В результате применения фильтра получаем упрощённую геометрическую модель. Её можно сохранить в формате VRML. На языке Python (https://www.python.org/) был разработан скрипт, который позволяет конвертировать данные из формата VRML в код APDL (ANSYS Parametric Design Language) для построения конечно-элементного разбиения, которое и использовалось для расчёта напряжённо-деформированного состояния кости.

На рис. 1 показан результат выполнения ANSYS-команд для построения геометрии бедренной кости различной сложности. Рис. 1 , а-результат применения фильтра QECD первый раз, на рис. 1, b и 1, c- результат его применения второй и третий раз соответственно. В дальнейшем для решения задач ползучести использовалась объёмная модель на рис. $1, b$, поскольку модель на рис. 1, с слишком детализирована и её использование в задачах ползучести на большие интервалы времени нецелесообразно из-за больших длительности расчёта.

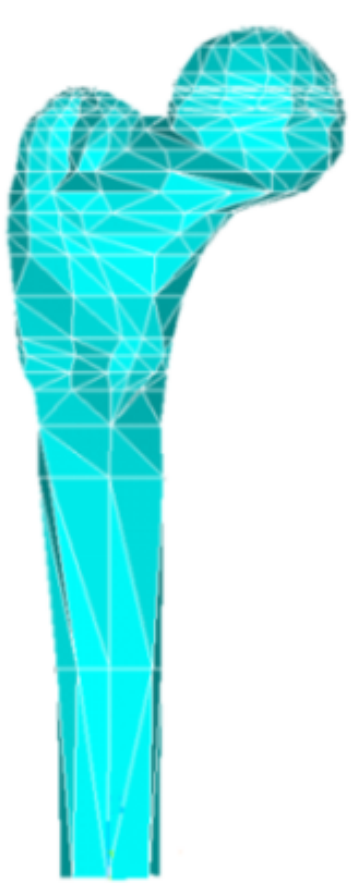

a

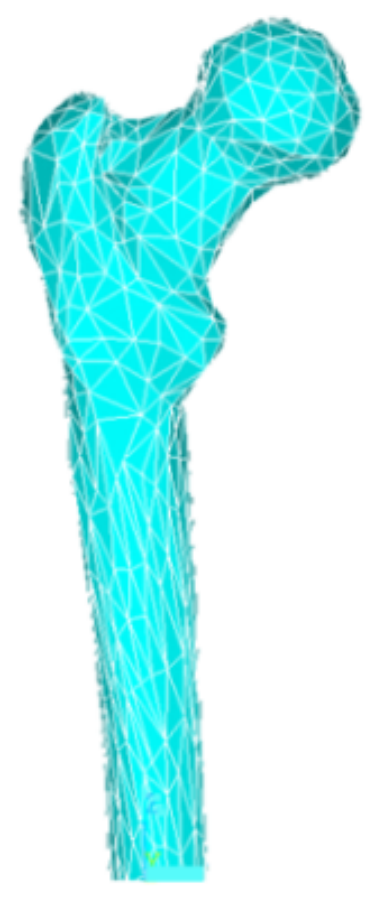

$b$

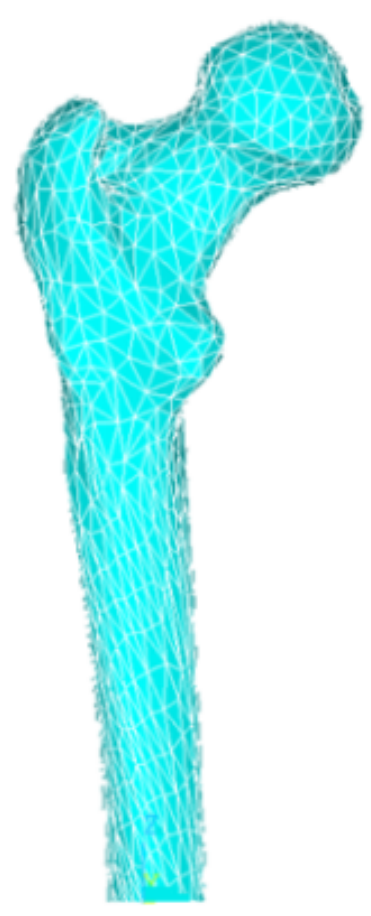

$c$

Рис. 1. Геометрия бедренной кости после применения фильтра QECD первый раз (a, использовано 379 точек), второй раз ( $b$, использовано 508 точек) и третий раз ( $c$, использовано 957 точек)

[Figure 1. The geometry of the femur after using the QECD filter.

Fig. $a$ is a case when the filter used first time (used 379 nodes).

Fig. $b$ is a case when the filter used second time (used 508 nodes).

Fig. $c$ is a case when the filter used third time (used 957 nodes)] 
Для построения двухслойной модели шейки бедренной кости из геометрической модели кости, полученной с использованием фильтра QECD, paзработана вспомогательная программа, которая преобразует исходную модель в двухслойную. Основная идея этой программы - создание внутреннего слоя кости за счёт уменьшения масштаба исходной геометрии с учётом невыпуклой особенности геометрии кости. Для построения двухслойной модели кости использовалась геометрическая модель, представленная на рис. 1, b. Разработан специальный метод, который изменяет координаты точек так, чтобы они оказались внутри внешнего слоя. Реализуется это путём умножения разницы координат и центра области на уменьшающий коэффициент, который представлен тремя компонентами, каждая из которых отвечает за изменение масштаба соответствующей оси координат. Так как кость невыпуклая, «экспериментально» выбрано несколько областей, в которых правила уменьшения разные. Всего таких областей получилось 7: головка, тело, несколько в шейке и большом вертеле. Правила уменьшения выбраны «экспериментально» таким образом, чтобы внутренний слой не выходил за наружный и расстояние от центра области до вновь полученной поверхности было на $10 \%$ меньше, чем расстояние до исходной поверхности. Полученный таким образом внутренний объём - это губчатая кость, а «тонкая» оболочка около него - кортикальная кость.

В настоящей работе при решении краевых задач в качестве имплантатов использовались только спица и спираль (винт-штопор), поскольку в работе [8] установлено, что у имплантата «шнековый винт» низкая эффективность.

На рис. 2 показаны разработанные геометрические модели для имплантатов типа «спица» и «винт-штопор», построенные в пакете ANSYS.

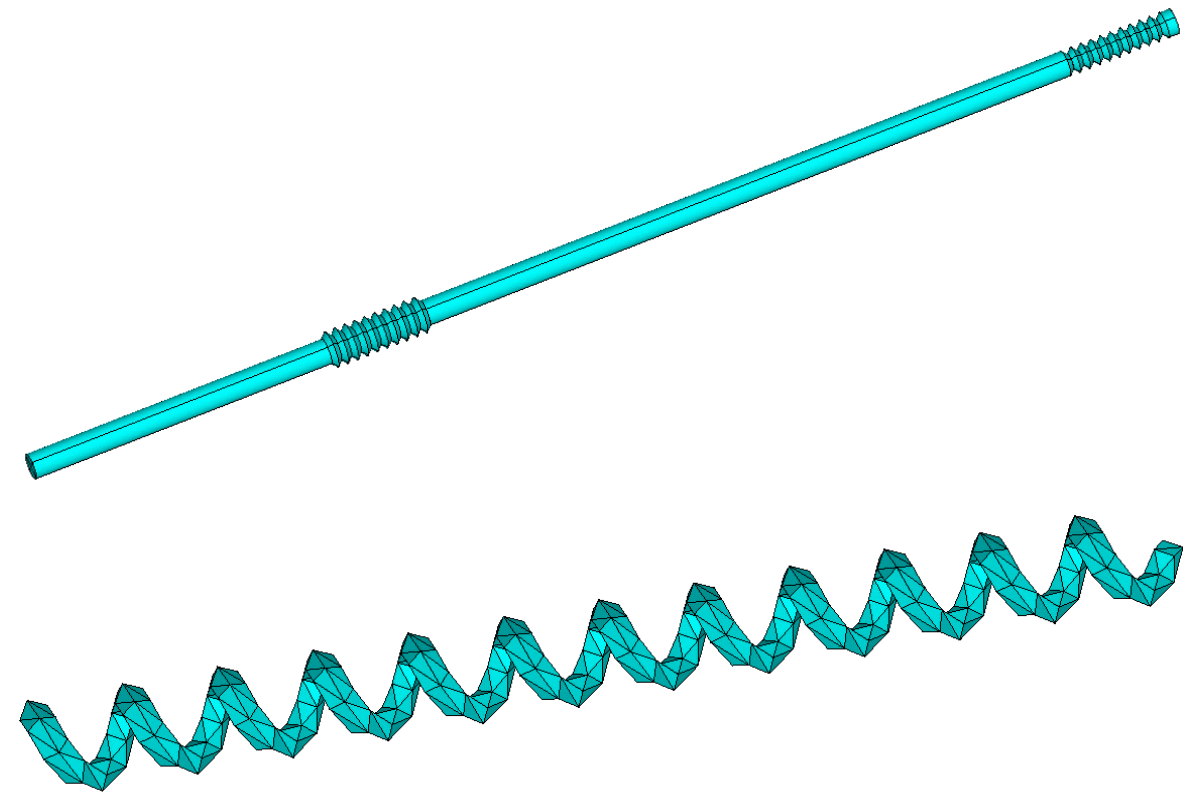

Рис. 2. Примеры геометрических моделей имплантатов типа «спица» (сверху) и типа «винт-штопор» (снизу), разработанных в ANSYS

[Figure 2. Examples of the geometric models developed in ANSYS for the spoke-type implant (top) and for the corkscrew-type implant (bottom)] 
Для автоматизации «внедрения» имплантатов в шейку бедренной кости человека и генерации конечно-элементной сетки разработаны вспомогательные программные средства со следующими особенностями:

- генерация APDL-команд для построения геометрии имплантатов по заданным характеристикам (длина, диаметр, шаг резьбы и прочее);

- позиционирование имплантата внутри шейки бедренной кости;

- генерация APDL-команд для объединения геометрий кости и имплантата;

- задание характеристик материала и типа конечных элементов для кости и имплантата.

Использованные в настоящей работе схемы позиционирования имплантатов показаны на рис. 3.

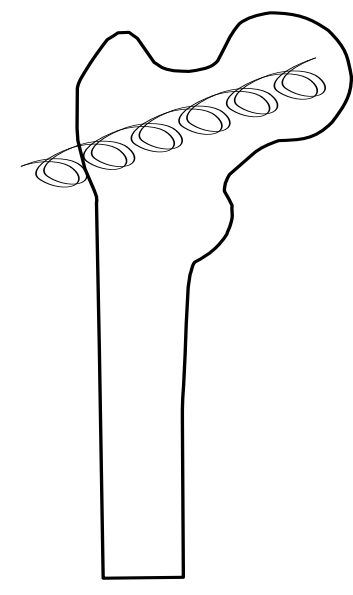

a

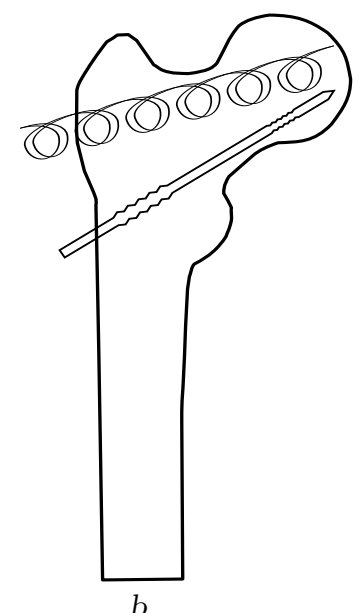

$b$

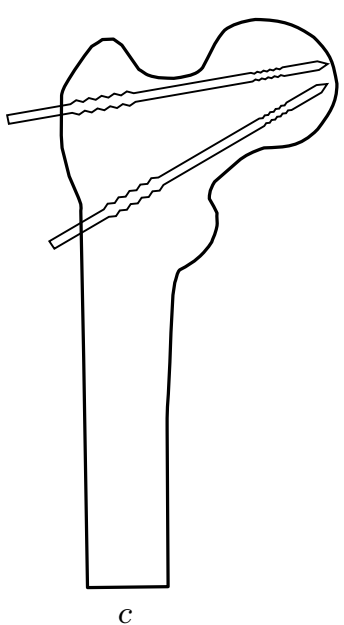

Рис. 3. Схемы систем армирования бедренной кости с использованием имплатнатов «винтштопор» $(\mathrm{a})$ «спица + винт-штопор» $(b)$ и «спица + спица» $(c)$

[Figure 3. Schemes of the femur reinforcements when used the corkscrew-type (a), the spoke-corkscrew-type $(b)$, and the spoke-spoke-type $(c)$ implants]

\section{2. Механические характеристики костной ткани и граничные условия.}

В работе моделируется напряжённо-деформированное состояние при длительных статических нагрузках, соответствующих хождению человека, т. е. напряжения в костной ткани далеки от предельных (критических). Поэтому, оставаясь в рамках механики сплошных сред, можно считать, что материал костная ткань находится в упругой области. Имеющиеся в научной литературе экспериментальные данные об упругих характеристиках компактной и губчатой костных тканей имеют существенный разброс данных, при этом они существенно зависят от возраста человека. Тем не менее в ряде работ выработаны надёжные экспериментальные методы определения эффективных упругих и реологических свойств костной ткани на уровне макромеханики сплошных сред [18-25], хотя во всех отмеченных работах указывается на недостаточную изученность механических характеристик этого биокомпозитного материала. При этом в работах [21-25] присутствуют совершенно противоположные мнения по поводу учёта анизотропии свойств в продольном и поперечном направлениях бедренной кости. Авторы настоящей работы используют в дальнейшем гипотезу анизотропии свойств как губчатой, так и компактной костной ткани. В соответствии с вышеизложенным при решении 
краевых задач использованы осреднённые величины упругих модулей (модулей Юнга) и коэффициентов Пуассона костной ткани и имплантата (титановый сплав), представленные в табл. 1.

Таблица 1

\section{Механические характеристики материалов [Mechanical properties of materials]}

\begin{tabular}{|c|c|c|}
\hline $\begin{array}{c}\text { Materials } \\
\text { Properties }\end{array}$ & $\begin{array}{c}\text { Descriptions } \\
\text { of Materials Properties }\end{array}$ & Values \\
\hline$E_{\mathrm{h}}$ & $\begin{array}{l}\text { Модуль упругости компактной костной ткани } \\
\text { The elastic modulus of the cortical bone tissue }\end{array}$ & $1.7 \cdot 10^{10} \mathrm{~Pa}$ \\
\hline$E_{\mathrm{c}}$ & $\begin{array}{l}\text { Модуль упругости губчатой костной ткани } \\
\text { The elastic modulus of the cancellous bone tissue }\end{array}$ & $3.25 \cdot 10^{8} \mathrm{~Pa}$ \\
\hline$E_{\mathrm{t}}$ & $\begin{array}{l}\text { Модуль упругости имплантата (титан) } \\
\text { The elastic modulus of the implant (the titanium alloy) }\end{array}$ & $1.1 \cdot 10^{11} \mathrm{~Pa}$ \\
\hline$\nu_{\mathrm{h}}$ & $\begin{array}{l}\text { Коэффициент Пуассона компактной костной ткани } \\
\text { The Poisson's ratio of the cortical bone tissue }\end{array}$ & 0.32 \\
\hline$\nu_{\mathrm{c}}$ & $\begin{array}{l}\text { Коэффициент Пуассона губчатой костной ткани } \\
\text { The Poisson's ratio of the cancellous bone tissue }\end{array}$ & 0.29 \\
\hline$\nu_{\mathrm{t}}$ & $\begin{array}{l}\text { Коэффициент Пуассона имплантата (титан) } \\
\text { The Poisson's ratio of the implant (the titanium alloy) }\end{array}$ & 0.32 \\
\hline$D$ & $\begin{array}{c}\text { Среднее расстояние между центрами структурных } \\
\text { единиц костной ткани } \\
\text { The average distance between the centers } \\
\text { of structural units of the bone tissue }\end{array}$ & $0.3 \mathrm{~mm}$ \\
\hline
\end{tabular}

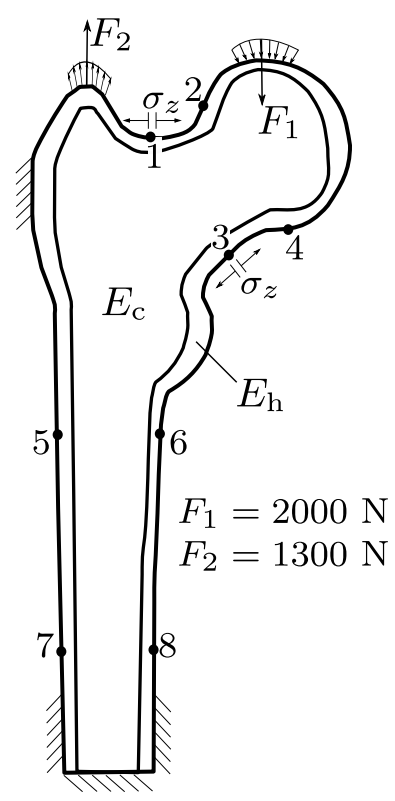

В настоящей работе условия для краевых задач ставились на основании работы [17], в которой моделировался процесс хождения человека. Согласно рекомендациям этой работы, граничные условия можно поставить исходя из схемы, представленной на рис. 4. На рис. 4 также приведены численные значения равнодействующих поверхностных сил $F_{1}$ и $F_{2}$, действующих на бедренную кость, и «критические точки» $1-8$, в которых выполнялся анализ напряжённо-деформированного состояния бедренной кости.

Рис. 4. Схема задания краевых условий для моделирования деформируемости бедренной кости при длительных нагрузках в условиях ползучести (в точках 1-8 проводилось исследование напряженно-деформированного состояния)

[Figure 4. The scheme with boundary conditions for the simulation of the deformability of the femur due to long time loads in creep conditions. $F_{1}$ and $F_{2}$ are the resultant forces acting on the bone. The points marked with numbers are studied points of the stress-strain state for the femur bone]

3. Построение феноменологической модели ползучести костной ткани. Для исследования деформируемости костной ткани при длительных нагрузках одной из основных задач является построение феноменологической теории ползучести. Поскольку для анализа напряжённо-деформированного состо- 
яния в армированной и неармированной шейке бедра использовался пакет ANSYS, теория ползучести выбиралась из библиотеки этого пакета. Как уже отмечалось, при ходьбе нагрузки незначительны и они далеки от предельных критических нагрузок. В связи с этим для построения одноосной модели ползучести были использованы реальные экспериментальные данные из работы [11], представленные точками на рис. 5. Они получены при напряжениях растяжения $\sigma_{0}=35.71 \mathrm{MПа} \mathrm{и} \sigma_{0}=53.56$ МПа, которые соответствуют упругой области костной ткани и составляют значения 0.28 и 0.42 от величины разрушающего напряжения при кратковременном нагружении (по данным работы [11]). Анализ экспериментальных данных (см. рис. 5) показывает, что они хорошо описываются классической теорией упрочнения [26]. Основной вариант неизотермической теории упрочнения в программном комплексе ANSYS для одноосного случая имеет вид

$$
\dot{p}=C_{1} \sigma^{C_{2}} p^{C_{3}} \exp \left(-C_{4} / T\right),
$$

где $C_{1}, C_{2}, C_{3}, C_{4}$ - коэффициенты модели ползучести; $p$ - деформация ползучести; $\sigma$ - напряжение; $T$ - температура.

Рис. 5. Экспериментальные (линии 3, 4) и расчетные (линии 1,2 ) кривые одноосной ползучести костной ткани, полученные по теории упрочнения: точки-экспериментальные данные из [11]; штриховые линии - кусочно-линейные аппроксимации экспериментальных данных; линии 1, 3 соответствуют растягивающему напряжению $\sigma_{0}=35.71$ МПа; линии 2,4 соответствуют растягивающему напряжению $\sigma_{0}=53.56 \mathrm{MПа}$

[Figure 5. The experimental (lines 3,4) and calculated $(1,2)$ curves of the uniaxial creep of bone tissue, obtained by hardening theory. The points are experimental datas from [11]; the dashed lines are piecewise-linear approximations for experimental datas; the lines marked with numbers 1 and 3 correspond to the tensile stress $\sigma_{0}=35.71 \mathrm{MPa}$; the lines marked with numbers 2 and 4 correspond to the tensile stress $\left.\sigma_{0}=53.56 \mathrm{MPa}\right]$

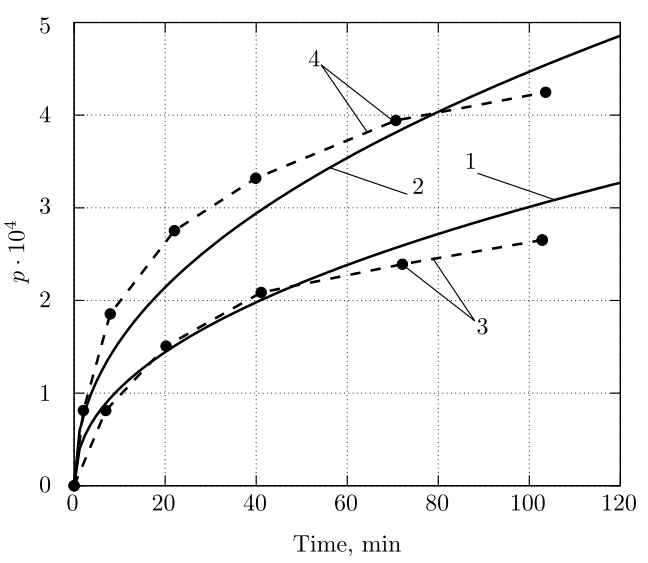

Поскольку температура костной ткани является постоянной (определяется температурой тела человека), в модели (1) величина $C_{4}=0$, и мы получаем трёхпараметрический вариант изотермический теории упрочнения. $\mathrm{K}$ сожалению, единой универсальной методики идентификации параметров модели (1) не имеется, поэтому для их идентификации разработана авторская методика.

Для определения коэффициентов модели ползучести выполним следующие действия. Считая $C_{4}=0$, проинтегрируем (1) при постоянном напряжении $\sigma=\sigma_{0}=$ const:

$$
p(t)=\left(C_{1}\left(1-C_{3}\right)\right)^{\frac{1}{1-C_{3}}} \sigma_{0}^{\frac{C_{2}}{1-C_{3}}} t^{\frac{1}{1-C_{3}}} .
$$

Введём следующие обозначения (как и в классической теории упрочнения [26]):

$$
-C_{3}=\alpha, \quad m=\frac{1}{(1+a)} .
$$


Тогда (2) принимает вид

$$
p(t)=\left(\frac{C_{1}}{m}\right)^{m} \sigma_{0}^{C_{2} m} t^{m} .
$$

Для определения параметра $m$ на одной и той же кривой берутся 2 точки $t_{1}$ и $t_{2}\left(t_{1} \neq t_{2}\right)$ и составляется соотношение

$$
\frac{p\left(t_{2}\right)}{p\left(t_{1}\right)}=\left(\frac{t_{2}}{t_{1}}\right)^{m},
$$

откуда

$$
m=\ln \frac{p\left(t_{2}\right)}{p\left(t_{1}\right)} / \ln \frac{t_{2}}{t_{1}} .
$$

Здесь $p\left(t_{1}\right)$ и $p\left(t_{2}\right)$ - значения деформации ползучести, соответствующие значениям времени $t_{1}$ и $t_{2}$ соответственно. Процедуру расчёта параметра $m$ по формуле (4) следует применить к разным точкам $t$ разных кривых $\sigma_{0}^{(i)}(i=$ $=1,2)$. Затем необходимо осреднить полученный спектр значений $m$ и полученную величину взять в качестве искомого значения этого параметра.

Для определения параметра $C_{2}$ выберем конкретное значение времени $t$ и два разных значения $\sigma_{0}^{(1)}$ и $\sigma_{0}^{(2)}$, тогда из $(3)$ получим

$$
\frac{p\left(\sigma_{0}^{(2)}, t\right)}{p\left(\sigma_{0}^{(1)}, t\right)}=\left(\frac{\sigma_{0}^{(2)}}{\sigma_{0}^{(1)}}\right)^{C_{2} m}
$$

откуда определим величину $C_{2}$ :

$$
C_{2}=\frac{1}{m} \ln \frac{p\left(\sigma_{0}^{(2)}, t\right)}{p\left(\sigma_{0}^{(1)}, t\right)} / \ln \frac{\sigma_{0}^{(2)}}{\sigma_{0}^{(1)}} .
$$

Здесь используется значение параметра $m$, определенное ранее на основе осреднения. Процедуру вычисления параметра $C_{2}$ по формуле (5) необходимо применить для разных $t$ и разных отношений $\sigma_{0}^{(i)} / \sigma_{0}^{(k)}(i, k=1,2 ; i \neq k)$ и осреднить полученные результаты. Таким образом, будет найдено окончательное осредненное значение $C_{2}$.

Когда известны величины $m$ и $C_{2}$, величину $C_{1}$ можно определить из $(3)$ :

$$
C_{1}=m\left(\frac{p(t)}{\sigma_{0}^{C_{2} m} t^{m}}\right)^{-m} .
$$

Процедуру вычисления параметра $C_{1}$ по формуле (6) необходимо применить для разных $t$ и $\sigma_{0}^{(i)}$ и провести осреднение полученных значений. В результате будем иметь окончательное осреднённое значение параметра $C_{1}$.

Для автоматизации процедуры определения коэффициентов $C_{1}, C_{2}, C_{3}$ (применения методики для различных кривых и различных точек кривых ползучести) разработана специальная программа и получены следующие параметры модели (1):

$$
C_{1}=1.1218 \cdot 10^{-28} ; \quad C_{2}=2.1351 ; \quad C_{3}=-1.1883 ; \quad C_{4}=0 .
$$


Результаты построения расчётных кривых ползучести костной ткани по модели (1) при постоянных напряжениях и найденных значениях (7), т. е. по формуле (2), приведены на рис. 5 сплошными линиями. В целом соответствие расчётных и экспериментальных данных удовлетворительное. Отклонение расчётных данных деформации ползучести $p$ от экспериментальных значений $\widetilde{p}$ вычисляется по формуле

$$
\Delta=\sqrt{\frac{1}{2} \sum_{i=1}^{2}\left(\frac{\sum_{k=1}^{n}\left(p^{i}\left(t_{k}\right)-\widetilde{p}^{i}\left(t_{k}\right)\right)^{2}}{\sum_{k=1}^{n}\left(\widetilde{p}^{i}\left(t_{k}\right)\right)^{2}}\right)} \cdot 100 \%
$$

где $i=1,2$ соответствует кривым ползучести при $\sigma_{0}=35.71 \mathrm{MПа}(i=1)$ и $\sigma_{0}=53.56 \mathrm{MПа}(i=2) ; t_{k}$ - значения времени, в которых вычисляются значения деформации ползучести $p^{i}\left(t_{k}\right)$ и $\widetilde{p}^{i}\left(t_{k}\right)$ на обеих кривых; $n=6-$ общее число экспериментальных точек. Расчёт по формуле (8) даёт значение $\Delta=17.9 \%$, которое можно считать удовлетворительным для теории ползучести [26].

4. Численное решение задачи реологического деформирования бедренной кости при длительных статических нагрузках. Решение краевой задачи со столь сложной границей области интегрирования может быть выполнено только численными методами. В качестве инструментария выбран метод конечных элементов (KЭ) программного комплекса ANSYS. Основной проблемой является выбор линейного размера КЭ. В работах $[27,28]$ показано, что костная ткань является многоступенчатым биокомпозитным материалом, причём характерные линейные размеры «блоков» на каждом масштабном уровне изменяются от нескольких десятков ангстрем до нескольких сотен микрометров. Однако строго математически рассматривать костную ткань как многофазный и многоступенчатый биокомпозитный материал не представляется возможным в силу отсутствия надёжных экспериментальных данных механических характеристик структурных элементов на каждом масштабном уровне. Поэтому в данной работе и предложено рассматривать костную ткань на уровне макромеханики сплошных сред, а согласно $[27,28]$ минимальный размер - расстояние между центрами остеонов, - позволяющий остаться в рамках механики сплошных сред, имеет величину $D \cong 0.3$ мм, которую в дальнейшем использовали как минимально допустимую величину линейного размера конечного элемента (см. табл. 1).

Для сравнительного анализа влияния времени выдержки на кинетику напряжённо-деформированного состояния в костной ткани в условиях ползучести расчёты выполнялись для временных интервалов 4 часа, 30 дней и 1 год. Время $t=4$ часа интегрально соответствует 8 часовой ходьбе с попеременным переставлением ног и последующим 16 часовым отдыхом (для ног), т. е. это суточная нагрузка на бедренную кость.

В качестве примера проанализируем результаты расчёта длительностью в 1 год (промежуточные интервалы 4 часа и 30 дней включены в этот временной промежуток). Результаты привязаны к локальным системам координат в каждой исследуемой точке (см. рис. 4). Ось $O Z$ всегда направлена по касательной к профилю сечения, ось $O Y$ - по нормали к контуру сечения (внутрь бедренной кости), ось $O X$ - таким образом, чтобы система координат $O X Y Z$ была правой. Выполненный анализ результатов расчёта в условиях длитель- 
ного нагружения в 1 год показал, что происходит существенная релаксация наибольшей (по модулю) компоненты тензора напряжений в критических областях (в областях, где возможно зарождение разрушения кости при «мгновенных» нагрузках), причём релаксация в армированной шейке бедра происходит более интенсивно, чем в неармированной. В качестве примера на рис. 6 показана зависимость максимального напряжения $\sigma_{z}(t)$ в локальных системах координат для имплантатов в «критических точках 1 и 3 » (см. нумерацию точек на рис. 4), из которого следует, что вследствие ползучести напряженность в наиболее опасных областях за счёт армирования снижается более чем на $18 \%$, что ведёт к менее вероятному перелому шейки бедра. Стоит отметить, что наиболее эффективной системой по отношению к величине скорости релаксации в этом исследовании также оказалась система «кость - спица + спица».

Это важный с точки зрения практики результат, поскольку армирование ускоряет процесс релаксации напряжений вследствие ползучести при длительной временной нагрузке (типа веса тела), т. е. происходит разгрузка наиболее нагруженных областей естественным образом. Поэтому, если в какой-то момент времени будут воздействовать «мгновенные» нагрузки (падение человека), то напряжённое состояние от них будет уже накладываться на «рабочие» остаточные напряжения после упругой разгрузки от повседневных нагрузок, а они с течением времени уменьшаются.

В табл. 2 приведены результаты исследования для всех точек, изображённых на рис. 4 (номера точек приведены в головке таблицы). Строки « $\sigma_{z}^{e}$ это напряжения $\sigma_{z}^{e}=\sigma_{z}(0)$ при решении чисто упругой задачи после приложения внешней нагрузки (т. е. при равномерно распределённой нагрузке с

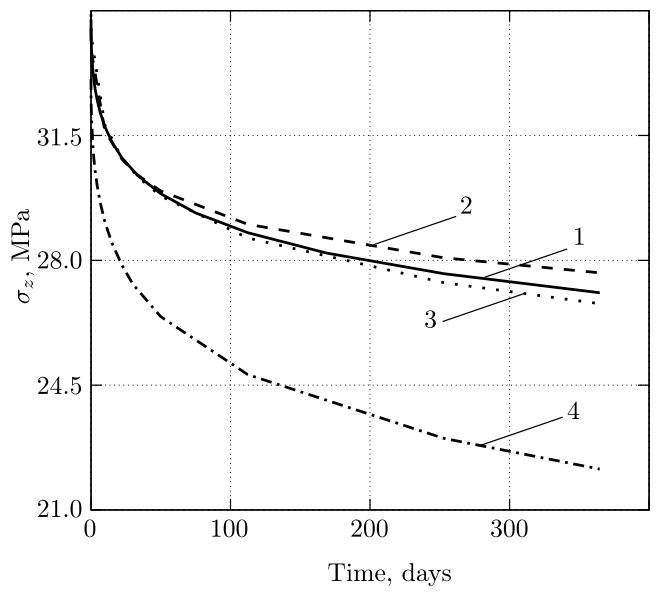

a

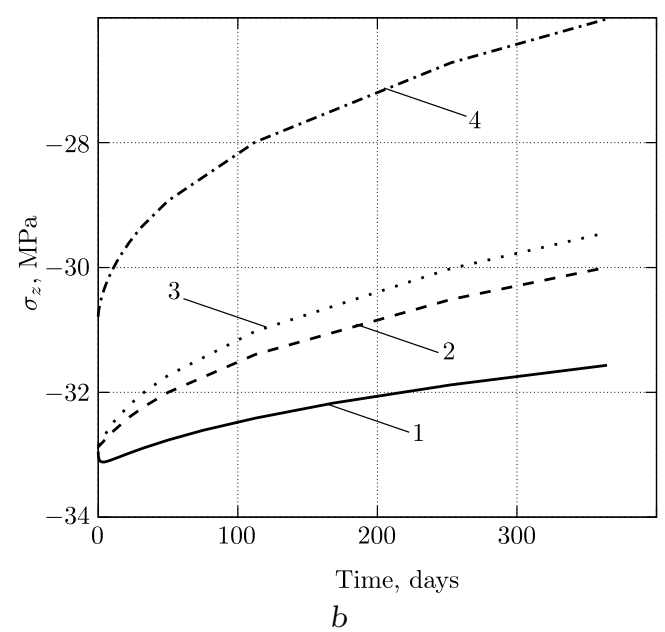

Рис. 6. Кинетика напряжений $\sigma_{z}(t)$ в «критической точке $1 »(a)$ и «критической точке 3» $(b)$ вследствие ползучести: кривые 1 - неармированная кость; кривые 2 -армирование «винт-штопор»; кривые 3 - армирование «винт-штопор + спица»; кривые 4 - армирование «спица + спица»

[Figure 6. Kinetics of the stress $\sigma_{z}(t)$ at the "critical points 1" (a) and the "critical points 2" (b) due to creep. The curves marked with 1 correspond to a unreinforced bone; the curves marked with 2 correspond to a reinforced bone by the corkscrew-type implant; the curves marked with 3 correspond to a reinforced bone by the spoke-corkscrew-type implant; the curves marked with 4 correspond to a reinforced bone by the spoke-spoke-type implant] 
равнодействующими $F_{1}$ и $F_{2}$, см. рис. 4$)$. В строках « $\Delta \sigma_{z} »$ приведены значения величины

$$
\Delta \sigma_{z}=\frac{\left|\sigma_{z}^{e}-\sigma_{z}^{c}\right|}{\left|\sigma_{z}^{c}\right|} \cdot 100 \%
$$

где $\sigma_{z}^{e}$ - напряжение, соответствующее упругому решению; $\sigma_{z}^{c}$ - напряжение, полученное в процессе релаксации вследствие ползучести к моменту времени $t_{c}$, т. е. $\sigma_{z}^{c}=\sigma_{z}\left(t_{c}\right)$, где $t_{c}$ принимает значения 4 ч., 30 дней или 1 год.

Значение $\Delta \sigma_{z}$ характеризует степень релаксации напряжений, которая протекает естественным образом при естественных нагрузках, действующих на человека. Как следует из данных табл. 2, наибольшая релаксация наблюдается в системе «кость - спица + спица» в самой опасной точке 1 и составляет величину около $49 \%$. Стоит отметить, что напряжения в теле бедренной кости (точки 6, 7, 8 на рис. 4) выше, чем в шейке, и почти не снижаются, однако тело бедренной кости не так сильно «страдает», как шейка бедра.

Таким образом, получен неожиданный результат - при превентивном армировании происходит снижение концентрации напряжений при мгновенном

Таблица 2

Значения напряжений $\sigma_{z}^{e}$ для упругой задачи (без учёта ползучести) и процентное отношение величины релаксации $\Delta \sigma_{z}$ в «критических точках» [The values of the stress $\sigma_{z}^{e}$ for the elastic stress problem (without creep) and the percentage of the stress relaxation value $\Delta \sigma_{z}$ at the "critical points"]

\begin{tabular}{|c|c|c|c|c|c|c|c|c|}
\hline \multirow{2}{*}{$\begin{array}{l}\text { Решение } \\
\text { [Solution] }\end{array}$} & \multicolumn{8}{|c|}{$\begin{array}{l}\text { Номера «критических точек» (см. рис. 4) } \\
\text { [The labels (numbers) of the "critical points" (see Fig. 4)] }\end{array}$} \\
\hline & 1 & 2 & 3 & 4 & 5 & 6 & 7 & 8 \\
\hline \multicolumn{9}{|c|}{ Неармированная кость [The case of a unreinforced bone] } \\
\hline$\sigma_{z}^{e}$, elastic, $\mathrm{MPa}$ & 34.8 & -19.2 & -32.9 & -16.3 & 34.5 & -40.4 & 41.9 & -49.5 \\
\hline$\Delta \sigma_{z}$ after 4 hours, $\%$ & 2 & 0 & 0 & 0 & 0 & 0 & 0 & 0 \\
\hline$\Delta \sigma_{z}$ after 30 days, $\%$ & 14 & 9 & 0 & 4 & 1 & 2 & -1 & 1 \\
\hline$\Delta \sigma_{z}$ after 365 days, $\%$ & 28 & 31 & 4 & 11 & 5 & 8 & 1 & 3 \\
\hline
\end{tabular}

Армирование «винт-штопор» [The case of a reinforced bone by the corkscrew-type implant]

\begin{tabular}{|c|c|c|c|c|c|c|c|c|}
\hline$\sigma_{z}^{e}$, elastic, MPa & 34.5 & -19.2 & -32.8 & -16.1 & 34.4 & -40.7 & 41.6 & -49.2 \\
$\Delta \sigma_{z}$ after 4 hours, \% & 2 & 0 & 0 & 0 & 0 & 0 & 0 & 0 \\
$\Delta \sigma_{z}$ after 30 days, \% & 13 & 10 & 2 & 3 & 1 & 1 & 0 & 2 \\
$\Delta \sigma_{z}$ after 1 year, \% & 25 & 38 & 9 & 9 & 6 & 7 & 1 & 5 \\
\hline
\end{tabular}

Армирование «винт-штопор + спица» [The case of a reinforced bone by the spoke-corkscrew-type implant]

\begin{tabular}{|c|c|c|c|c|c|c|c|c|}
\hline$\sigma_{z}^{e}$, elastic, MPa & 34.9 & -19.4 & -32.8 & -16.0 & 34.2 & -40.0 & 41.7 & -48.9 \\
$\Delta \sigma_{z}$ after 4 hours, \% & 2 & 1 & 0 & 0 & 0 & 0 & 0 & 0 \\
$\Delta \sigma_{z}$ after 30 days, \% & 14 & 12 & 2 & 4 & 0 & 0 & -1 & 3 \\
$\Delta \sigma_{z}$ after 1 year, \% & 30 & 41 & 12 & 15 & 2 & 4 & 0 & 6 \\
\hline
\end{tabular}

Армирование «спица + спица» [The case of a reinforced bone by the spoke-spoke-type implant]

\begin{tabular}{|c|c|c|c|c|c|c|c|c|}
\hline$\sigma_{z}^{e}$, elastic, MPa & 33.1 & -19.3 & -30.8 & -15.4 & 31.0 & -33.2 & 29.5 & -36.0 \\
$\Delta \sigma_{z}$ after 4 hours, \% & 2 & 1 & 0 & 0 & 0 & 0 & 0 & 0 \\
$\Delta \sigma_{z}$ after 30 days, \% & 21 & 13 & 5 & 6 & -1 & 0 & 0 & 1 \\
$\Delta \sigma_{z}$ after 1 year, \% & 49 & 46 & 18 & 23 & -1 & 3 & 3 & 3 \\
\hline
\end{tabular}


нагружении бедренной кости [5,8], а далее в условиях длительного статического нагружения естественным образом (вследствие свойства ползучести костной ткани) происходит дополнительная существенная разгрузка (релаксация напряжений) уже имеющегося напряжённого состояния в шейке бедра, сформированного в момент приложения нагрузки. Связано это с тем, что титан в этих условиях (температура тела человека) не обладает свойствами ползучести и напряжения из костной ткани «перекачиваются» в имплантат. Отсюда можно сделать вывод, что временная составляющая ни в коей мере не ухудшает положительный эффект от снижения концентрации напряжений за счёт армирования, что, несомненно, является позитивным моментом с точки зрения медицинской практики.

\section{Выводы по работе.}

1. Разработана математическая модель оценки влияния армирующих элементов на деформирование бедренной кости при длительных статических нагрузках в условиях ползучести.

2. Построена феноменологическая модель ползучести компактной костной ткани на основе теории упрочнения, предложена методика идентификации её параметров и выполнена проверка адекватности экспериментальным данным.

3. Разработан и реализован численный метод решения краевой задачи ползучести бедренной кости с различными армирующими элементами в условиях длительного стационарного нагружения. Выполненный анализ показал, что происходит существенная релаксация напряжений в наиболее нагруженных областях вследствие ползучести, причём в армированной шейке бедра она происходит более интенсивно, чем в неармированной. Так, для имплантата «спица + спица» при длительности нагружения в течение 1 года при естественных нагрузках, соответствующих хождению человека, напряжённость в наиболее нагруженной области шейки бедра вследствие ползучести снижается на 49 \% по отношению к напряжённости в начальный момент приложения нагрузки.

4. Временная составляющая (длительная стационарная нагрузка) ни в коей мере не ухудшает положительный эффект от снижения концентрации напряжений за счёт армирования шейки бедра, что является позитивным моментом с точки зрения медицинской практики.

\section{ORCIDs}

Владимир Павлович Радченко: http://orcid.org/0000-0003-4168-9660

Анатолий Вадимович Нехожин: http://orcid.org/0000-0002-6867-1041

\section{БИБЛИОГРАФИЧЕСКИЙ СПИСОК}

1. Матвеев А. Л. Оперативный способ профилактики переломов шейки бедренной кости: Патент РФ на на изобретение № 2316280 от 10.02.2008.

2. Матвеев А. Л., Нехожин А. В. Устройство для армирования шейки бедренной кости u превентивной профилактики переломов: Патент РФ на на изобретение № 98901 от 10.11.2010.

3. Матвеев А. Л., Нехожин А. В., Минасов Т. Б., Фролов А. В. Устройство для армирования кости и профилактики переломов ее при остеопорозе: Патент РФ на на изобретение № 121725 от 10.11.2012.

4. Матвеев А. Л., Нехожин А. В., Минасов Т. Б., Степанов О. Н., Дубров В. Э. Устрой- 
ство для профилактического армирования и предупреждения переломов проксималъного отдела бедра: Патент РФ на на изобретение № 136703 от 20.01.2014.

5. Радченко В. П., Нехожин А. В., Матвеев А. Л. Математическое моделирование напряжённого состояния армированной костной ткани шейки бедра при статических нагрузках// Вестн. Сам. гос. техн. ун-та. Сер. Физ.-мат. науки, 2011. № 4(25). С. 75-81. doi : $10.14498 / v s g t u 1037$.

6. Афанасьев Ю. И., Юрина Н. А., Котовский Е. Ф. Гистология. М.: Медицина, 2002. 774 c.

7. Рогожников Г. И., Конюхова С. Г., Няшин Ю. И., Чернопазов С. А., Еремина С. В. Влияние модуля упругости губчатой и кортикальной кости на напряженное состояние в области пластинчатого имплантата при окклюзионной нагрузке // Российский журнал биомеханики, 2004. Т. 8, № 1. С. 54-60.

8. Нехожин А. В. Двухслойная математическая модель шейки бедра человека для исследования напряжённого состояния при армировании имплантатами различной конструкции // Вестн. Сам. гос. техн. ун-та. Сер. Физ.-мат. науки, 2013. № 3(32). С. 129135. doi : $10.14498 / v s g t u 1254$.

9. Минасов Т. Б., Матвеев А. Л., Нехожин А. В. Прочностные характеристики проксимального отдела бедренной кости в условиях внутреннего силового шунтирования // Вестн. Сам. гос. техн. ун-та. Сер. Физ.-мат. науки, 2013. № 4(33). С. 98-106. doi : $10.14498 /$ vsgtu 1270 .

10. Минасов Б. Ш., Ханин М. Ю., Якупов Р. Р., Минасов Т. Б. Результаты стендовых испытаний системы кость-имплантат-кость в условиях стандартного остеосинтеза при переломах проксимального отдела бедра // Казанский медицинский журнал, 2010. № 1. C. 40-44.

11. Кнетс И. В., Вилкс Ю. К. Ползучесть компактной костной ткани человека при растяжении // Механика композитных материалов, 1975. № 4. С. 634-638.

12. Мелнис А. Э., Кнетс И. В., Моорлат П. А. Особенности деформирования компактной костной ткани человека при ползучести в условиях растяжения // Механика композитных материалов, 1979. № 5. С. 861-867.

13. Мелнис А. Э., Лайзан Я. Б. Нелинейная ползучесть компактной костной ткани человека при растяжении // Механика полимеров, 1978. №1. С. 97-100.

14. Радченко В. П., Шапиевский Д. В. Математическая модель ползучести микронеоднородного нелинейно-упругого материала // ПМТФ, 2008. Т. 49, № 3. С. 157-163.

15. Harlan N. Titanium Bone Implants // Materials Technology, 2000. vol. 15, no. 3. pp. $185-187$.

16. Harlan N., Reyes R., Bourell D. L., Beaman J. J. Titanium Castings using Laser Scanned Data and Selective Laser Sintered Zirconia Molds // Journal of Materials Engineering and Performance, 2001. vol. 10, no. 4. pp. 410-413. doi: 10.1361/105994901770344818.

17. Harlan N., Reyes R., Bourell D. L. Building Better Bones // Foundry Management E Technology, 2000. vol. 128, no. 8. pp. 82-83.

18. Лайуни Р. К вопросу о механических свойствах костной ткани / Физическое воспитание студентов творческих специальностей. Т. 4. Харьков, 2002. С. 18-22.

19. Brown C., Norman T. L., Kish V. L., Blaha J. D. Transverse Creep Response in Human Femoral Bone / American Society of Biomechanics, 1999. pp. 164-165, Available at http: //works.bepress.com/timothy_norman/123/ (September 18, 2015).

20. Reilly D. T., Burstein A. H. The elastic and ultimate properties of compact bone tissue // Journal of Biomechanics, 1975. vol.8, no.6. pp. 393-405. doi:10.1016/0021-9290(75) 90075-5.

21. Svesnsson N. L., Valliappan S., Wood R. D. Stress analysis of human femur with implanted charnley prosthesis // Journal of Biomechanics, 1977. vol. 10, no.9. pp. 581-588. doi: 10. 1016/0021-9290(77)90038-0.

22. Huiskes R., Janssen J. D., Slooff T. J. A detailed comparison of experimental and theoretical stress analyses of a human femur / Mechanical Properties of Bone. vol. 45; ed. S. C. Owin. New York: ASME, 1981. pp. 211-234. 
23. Lang S. B. Elastic coefficients of animal bone// Science, 1969. vol. 165, no. 3890. pp. 287288. doi : 10.1126/science.165.3890.287.

24. Van Buskirk W. C., Ashman R. B. The elastic moduli of bone/ Mechanical Properties of Bone. vol. 45; ed. S. C. Owin. New York: ASME, 1981. pp. 131-143.

25. Винц Х. Изменение механических свойств компактной костной ткани человека в зависимости от возраста // Механика полимеров, 1975. №11. С. 659-663.

26. Работнов Ю. Н. Ползучесть элементов конструкиий. М.: Наука, 1966. 752 с.

27. Утенькин А. А. Кость - многоэтажный композит // Химия и жизнь, 1981. № 4. С. $38-$ 40.

28. Минасов Т. Б., Стрижков А. Е., Бакусов Л. М., Насыров Р. В. Структурная самоорганизачия костной ткани и ее механизмы как источник диагностической информачии. Уфа, 2010. 116 c.

Поступила в редакцию 04/VII/2015;

в окончательном варианте - 18/IX/2015;

принята в печать $-24 / \mathrm{XI} / 2015$.

Vestn. Samar. Gos. Techn. Un-ta. Ser. Fiz.-mat. nauki

[J. Samara State Tech. Univ., Ser. Phys. \& Math. Sci.], 2015, vol. 19, no. 4, pp. 768-784

ISSN: 2310-7081 (online), 1991-8615 (print) doi: http://dx.doi.org/10.14498/vsgtu1441

MSC: $74 \mathrm{~L} 15$

\title{
MATHEMATICAL MODELING OF DEFORMATION OF REINFORCED FEMUR DURING PROLONGED STATIC LOADS
}

\section{P. Radchenko, A. V. Nekhozhin}

Samara State Technical University,

244, Molodogvardeyskaya st., Samara, 443100, Russian Federation.

\begin{abstract}
A two-layer mathematical model of a human femur neck reinforced implants of different design for modeling stress-strain state which occurs during a surgical procedure to prevent femur neck fractures by the forced introduction of metallic implants is proposed. Engineered implant designs are provided. Methods and software for geometric modeling of femur embedded with the implants are developed. New boundary value problems to evaluate kinetics in creep conditions of the stress-strain state of reinforced and non-reinforced femoral neck during prolonged static loads corresponding to human foot traffic are formulated. Effective elastic properties of cortical and cancellous bone, power and kinematic boundary value problems. A phenomenological
\end{abstract}

(C) 2015 Samara State Technical University.

Please cite this article in press as:

Radchenko V. P., Nekhozhin A. V. Mathematical modeling of deformation of reinforced femur during prolonged static loads, Vestn. Samar. Gos. Tekhn. Univ., Ser. Fiz.-Mat. Nauki [J. Samara State Tech. Univ., Ser. Phys. \& Math. Sci.], 2015, vol. 19, no. 4, pp. 768-784. doi: 10.14498/vsgtu1441. (In Russian)

Authors Details:

Vladimir P. Radchenko (Dr. Phys. \& Math. Sci.; radch@samgtu.ru, Corresponding Author), Head of Dept., Dept. of Applied Mathematics \& Computer Science.

Anatoliy V. Nekhozhin (stswoon@yandex.ru), Postgraduate Student, Dept. of Applied Mathematics \& Computer Science. 
creep model for compact bone tissue is constructed. The technique of identifying the parameters is developed. A check of its adequacy to experimental data is carried out. Based on the finite element method the numerical method for solving the provided boundary value problems at macro level of continuum mechanics is developed. A lot of variative calculations allowed developing recommendations for the rational positioning of the implant in order to minimize stress concentrations. The performed analysis showed that there is a significant relaxation of stresses in the most loaded areas due to creep. Relaxation is more intense in reinforced femoral neck than in the unreinforced. Thus the tension in the most loaded femoral neck area due to creep is reduced by $49 \%$ with respect to the intensity of the initial time of loading for femur which is reinforced by the spoke-spoke-type implant when loading duration is 1 year under natural loads corresponding to human foot traffic. It was found that the time component (long-term fixed load) does not impair the positive effect of reducing the stress concentration due to a femoral neck reinforcement which is a positive fact from the medical practice point of view.

Keywords: femur bone, implant, femoral neck, geometric modeling, finite element method, creep, stress-strain state, relaxation.

doi: http://dx.doi.org/10.14498/vsgtu1441

\section{ORCIDs}

Vladimir P. Radchenko: http://orcid.org/0000-0003-4168-9660

Anatoliy V. Nekhozhin: http://orcid.org/0000-0002-6867-1041

\section{REFERENCES}

1. Matveev A. L. Operative way of femoral neck fractures preventing, Invention patents of the Russian Federation. No. 2316280 of February 10, 2008 (In Russian).

2. Matveev A. L., Nekhozhin A. V. The device for the reinforcement of the femoral neck and the first fracture prevention, Invention patents of the Russian Federation. No. 98901 of November 10, 2010 (In Russian).

3. Matveev A. L., Nekhozhin A. V., Minasov T. B., Frolov A. V. The device for the reinforcement of the femoral neck and the fracture prevention for the osteoporosis, Invention patents of the Russian Federation. No. 121725 of November 10, 2012 (In Russian).

4. Matveev A. L., Nekhozhin A. V., Minasov T. B., Stepanov O. N., Dubrov V. É. The device for the preventive reinforcement and prevent proximal femur fractures, Invention patents of the Russian Federation. No. 136703 of January 20, 2014 (In Russian).

5. Radchenko V. P., Nekhozhin A. V., Matveev A. L. Mathematical modeling of the stress state of reinforced bone tissue of the femoral neck with static load, Vestn. Samar. Gos. Tekhn. Univ. Ser. Fiz.-Mat. Nauki [J. Samara State Tech. Univ., Ser. Phys. \& Math. Sci.], 2011, no. 4(25), pp. 75-81 (In Russian). doi: 10.14498/vsgtu1037.

6. Afanas'ev Yu. I., Yurina N. A., Kotovskiy E. F. Gistologiia [Histology]. Moscow, Meditsina, 2002, 774 pp. (In Russian)

7. Rogozhnikov G. I., Konyuhova S. G., Nyashin Yu. I., Chernopazov S. A., Eremina S. V. The influence of elasticity modulus of spongy and cortical bone on stress state near planar implant under occlusal load, Rossiiskii zhurnal biomekhaniki [Russian Journal of Biomechanics], 2004, vol. 8, no. 1, pp. 54-60 (In Russian).

8. Nekhozhin A. V. Bilayer mathematical model of human femur neck for research of the stress state after reinforcement with different designs of implants, Vestn. Samar. Gos. Tekhn. Univ. Ser. Fiz.-Mat. Nauki [J. Samara State Tech. Univ., Ser. Phys. \& Math. Sci.], 2013, no. 3(32), pp. 129-135 (In Russian). doi: 10.14498/vsgtu1254.

9. Minasov T. B., Matveev A. L., Nekhozhin A. V. Strength characteristics of the proximal femur in conditions of internal force shunting, Vestn. Samar. Gos. Tekhn. Univ. Ser. Fiz.- 
Mat. Nauki [J. Samara State Tech. Univ., Ser. Phys. \& Math. Sci.], 2013, no. 4(33), pp. 98106 (In Russian). doi: 10.14498/vsgtu1270.

10. Minasov B. Sh., Khanin M. Yu., Yakupov R. R., Minasov T. B. The results of bone-implantbone bench tests in standard osteosynthesis during fractures of the proximal part of the thigh bone, Kazanskii meditsinskii zhurnal [Kazan Medical Journal], 2010, no. 1, pp. 40-44 (In Russian).

11. Knets I. V., Vilks Yu. K. Creep of compact human bony tissue under tension, Polymer Mechanics, 1975, vol. 11, no.4, pp. 543-547. doi: 10.1007/bf00856779.

12. Melnis A. É., Knets I. V., Moorlat P. A. Deformation behavior of human compact bone tissue upon creep under tensile testing, Mechanics of Composite Materials, 1980, vol.15, no. 5, pp. 574-579. doi: 10.1007/BF00605581.

13. Melnis A. É., Laizan Ya. B. Nonlinear creep of human compact bone tissue upon stretching, Polymer Mechanics, 1978, vol. 14, no. 1, pp. 82-84. doi: 10.1007/BF00859565.

14. Radchenko V. P., Shapievskii D. V. Mathematical model of creep for a microinhomogeneous nonlinearly elastic material, Journal of Applied Mechanics and Technical Physics, 2008, vol. 49, no. 3, pp. 478-483. doi: 10.1007/s10808-008-0064-9.

15. Harlan N. Titanium Bone Implants, Materials Technology, 2000, vol. 15, no. 3, pp. 185-187.

16. Harlan N., Reyes R., Bourell D. L., Beaman J. J. Titanium Castings using Laser Scanned Data and Selective Laser Sintered Zirconia Molds, Journal of Materials Engineering and Performance, 2001, vol. 10, no. 4, pp. 410-413. doi: 10.1361/105994901770344818.

17. Harlan N., Reyes R., Bourell D. L. Building Better Bones, Foundry Management \& Technology, 2000, vol. 128, no. 8, pp. 82-83.

18. Liayouni R. To a question on mechanical charecteristics bone tissue, Fizicheskoe vospitanie studentov tvorcheskikh spetsial'nostei [Physical education of students of creative disciplines], vol. 4. Khar'kov, 2002, pp. 18-22 (In Russian).

19. Brown C., Norman T. L., Kish V. L., Blaha J. D. Transverse Creep Response in Human Femoral Bone, American Society of Biomechanics, 1999, pp. 164-165, Available at http: //works.bepress.com/timothy_norman/123/ (September 18, 2015).

20. Reilly D. T., Burstein A. H. The elastic and ultimate properties of compact bone tissue, Journal of Biomechanics, 1975, vol.8, no.6, pp. 393-405. doi:10.1016/0021-9290(75) 90075-5.

21. Svesnsson N. L., Valliappan S., Wood R. D. Stress analysis of human femur with implanted charnley prosthesis, Journal of Biomechanics, 1977, vol.10, no. 9, pp. 581-588. doi: 10 . 1016/0021-9290 (77) 90038-0.

22. Huiskes R., Janssen J. D., Slooff T. J. A detailed comparison of experimental and theoretical stress analyses of a human femur, Mechanical Properties of Bone, vol. 45; ed. S. C. Owin. New York, ASME, 1981, pp. 211-234.

23. Lang S. B. Elastic coefficients of animal bone, Science, 1969, vol. 165, no. 3890, pp. 287-288. doi: 10.1126/science.165.3890.287.

24. Van Buskirk W. C., Ashman R. B. The elastic moduli of bone, Mechanical Properties of Bone, vol. 45; ed. S. C. Owin. New York, ASME, 1981, pp. 131-143.

25. Vinz H. Change in the mechanical properties of human compact bone tissue upon aging, Polymer Mechanics, 1975, T. 11, № 4, C. 568-571. doi: 10.1007/bf00856785.

26. Rabotnov Yu. N. Creep problems in structural members. Amsterdam, London, NorthHolland Publ. Co., 1969, xiv+822 pp.

27. Uten'kin A. A. Bone is a multi-storey composite, Khimiya i Zhizn', 1981, no. 4, pp. 38-40 (In Russian).

28. Minasov T. B., Strizhkov A. E., Bakusov L. M., Nasyrov R. V. Strukturnaia samoorganizatsiia kostnoi tkani $i$ ee mekhanizmy kak istochnik diagnosticheskoi informatsii [Structural self-organization of bone tissue and its mechanisms as a source of diagnostic information]. Ufa, 2010, 116 pp. (In Russian)

Received 04/VII/2015;

received in revised form 18/IX/2015; accepted 24/XI/2015. 